\title{
Translocation of IRS-1 to Cytosol Attenuates Insulin-Stimulated Glucose Transport without Affecting PI3-Kinase Activity
}

\author{
Junko Kawahara, Isao Usui*, Tetsuro Haruta, Yukiko Kanatani, Kazuyuki Hiratani, Atsuko Takano, \\ Tatsuhito Uno, Minoru Iwata, Masashi Kobayashi and Kazuyuki Tobe
}

\author{
First Department of Internal Medicine, University of Toyama, Japan
}

\begin{abstract}
IRS-1 is a major substrate for insulin receptor tyrosine kinase. It is reported that intracellular translocation of serine-phosphorylated IRS-1 from low density microsome (LDM) fraction to cytosol attenuates its ability to transmit insulin signaling to the downstream molecules. In this study, we examined which insulin signal and action were affected by translocation of IRS-1 in 3T3-L1 adipocytes. Adenovirus-mediated overexpression of constitutively active PI3-kinase (p110CAAX) induces translocation of IRS-1 to cytosol without increasing IRS-1 tyrosine phosphorylation. IRS-1 protein localized in cytosol fraction in p110CAAX-expressing cells maintained the ability to be tyrosine-phosphorylated by short term insulin treatment. Long term treatment with insulin for 4 to $8 \mathrm{~h}$ decreased tyrosine phosphorylation of IRS-1, PI3kinase activity, Akt phosphorylation and glucose uptake by second stimulation with insulin. Pretreatment with rapamycin, a specific mTOR inhibitor, increased the protein level of IRS-1 in LDM fraction and restored the attenuated insulin signaling and glucose uptake after long term insulin treatment. On the other hand, pretreatment with lactacystin, a specific proteasomal inhibitor, increased the protein level of serine-phosphorylated IRS-1 in cytosol fraction. In this condition, insulin signaling from IRS-1 to Akt was restored, but glucose uptake was not. Taken together, we conclude that localization of IRS-1 in LDM fraction is necessary for insulin-stimulated glucose uptake, while IRS-1, once serinephosphorylated and translocated to cytosol, fails to stimulate glucose uptake despite its intact ability to be tyrosine phosphorylated and to transmit insulin signaling to Akt level.
\end{abstract}

Keywords: IRS-1, serine phosphorylation, mTOR, insulin resistance.

\section{INTRODUCTION}

Insulin receptor tyrosine kinase is activated in response to insulin stimulation. It phosphorylates the intracellular substrates on tyrosine residues, leading to stimulation of insulin actions. Insulin receptor substrate (IRS)-1 is the major substrate for insulin receptor tyrosine kinase in 3T3L1 adipocytes. Activation of phosphatidyl inositol (PI) 3kinase, recruited to the tyrosine-phosphorylated IRS-1, mediates various metabolic actions of insulin, such as translocation of GLUT4 followed by stimulation of glucose transport $[1,2]$. Akt is one of the signaling molecules downstream of PI 3-kinase and is involved in many PI3kinase-mediated insulin actions.

Several recent studies have reported that serine/threonine phosphorylation of IRS-1 is highly associated to the impaired insulin signaling [3-11]. For example, some studies reported that serine phosphorylation of IRS-1 impairs insulin-stimulated tyrosine phosphorylation of IRS-1 [3-8]. Other reports suggests that serine phosphorylation of IRS-1 somehow inhibits PI3-kinase activity, independently of IRS1 tyrosine phosphorylation level [9-11]. Serine/threonine phosphorylation of IRS-1 is linked with altered intracellular localization of IRS-1, which interferes with the interaction of IRS-1 with insulin receptor, resulting in the decreased

*Address correspondence to this author at the First Department of Internal Medicine, University of Toyama, 2630 Sugitani, Toyama, 930-0194, Japan; Tel: +81-76-434-7287; Fax: +81-76-434-5025;

E-mail: isaousui-tym@umin.ac.jp response of IRS-1 tyrosine phosphorylation and the downstream signaling events [12].

We previously reported that the activation of a pathway sensitive to rapamycin, an inhibiter of mammalian target of rapamycin (mTOR), was involved in serine/threonine phosphorylation and intracellular translocation of IRS-1 from low-density microsome (LDM) to cytosol after prolonged exposure of 3T3-L1 adipocytes to insulin. The activation of this pathway by longer insulin exposure then led to the proteasomal degradation of IRS-1, resulting in a physiological termination of insulin signaling [13, 14]. Recent reports also support this mTOR-dependent feedback mechanism [15-17]. They suggest that this mechanism may play a role in the pathophysiology of insulin resistance observed in genetic and diet-induced animal models [17]. However, in our previous study, IRS-1 kept its ability to be tyrosine-phosphorylated and to phosphorylate Akt even when it was serine/threonine phosphorylated and translocated to cytosol [13]. Because serine/threonine phosphorylation and translocation occurred after IRS-1 was already tyrosine-phosphorylated by insulin exposure, these studies could not clarify which function of IRS-1 was affected by its serine/threonine phosphorylation and translocation $[13,14]$.

To compare the function of IRS-1 with or without serine/threonine phosphorylation and translocation to cytosol, in the present study, we employed the cells expressing constitutively active form of PI3-kinase by using adenovirus vectors. In these cells, mTOR pathway was activated and IRS-1 
serine/threonine phosphorylation occurred without increasing tyrosine phosphorylation. We also employed the cells treated with either rapamycin or lactacystin, a proteasomal inhibitor, with prolonged insulin exposure. Rapamycin inhibits both IRS1 serine/threonine phosphorylation and its translocation to cytosol. On the other hand, lactacystin merely inhibits proteasomal degradation of IRS-1 but it does not affect its serine/threonine phosphorylation and translocation to cytosol. Using these cell culture systems, we could examine the effects of serine/threonine phosphorylation and translocation of IRS-1 on its functions. Here, we show that glucose transport is attenuated likely through the translocation of IRS-1, despite intact abilities to be tyrosine-phosphorylated and to activate Akt.

\section{MATERIALS AND METHODOLOGY}

\section{Materials}

Dulbecco's modified Eagle's medium (DMEM) and fetal calf serum (FCS) were purchased from GibcoBRL, Life Technologies (Gaithersburg, MD). [1, 2- $\left.{ }^{3} \mathrm{H}\right]$ 2-deoxyglucose (DOG) was purchased from NEN Life Science Products (Boston, MA). The Lilly Research Laboratories (Indianapolis, IN) kindly provided porcine insulin. Horseradish peroxidase (HRP)-conjugated monoclonal anti-phosphotyrosine antibody $(\mathrm{PY} 20 \mathrm{H})$ was purchased from Transduction Laboratories (Lexington, KY). Anti-IRS-1 and anti-phospho-Ser ${ }^{307}$-IRS-1 antibodies were purchased from Upstate Biotechnology (Lake Placid, NY). Phosphospecific antibodies against Akt and antiphospho-Ser ${ }^{612}$-IRS-1 antibody were purchased from Cell Signaling Technology (Beverly, MA). Rapamycin was purchased from Sigma (St. Louis, MO). Lactacystin was purchased from Calbiochem (La Jolla, CA). Electrophoresis reagents were from Bio-Rad (Hercules, CA). All other reagents were from standard suppliers.

\section{Cells}

Murine 3T3-L1 preadipocytes were purchased from American Type Culture Collection (ATCC) (Rockville, MD). Cells were cultured, maintained, and differentiated essentially as described previously $[13,14]$. Human embryonic kidney 293 cells obtained from ATCC were cultured in DMEM containing $10 \% \mathrm{FCS}$ in an atmosphere of $5 \% \mathrm{CO}_{2}$.

\section{Infection of Recombinant Adenovirus Vectors}

The recombinant adenovirus type 5 (Ad5)-p110 CAAX containing bovine $\mathrm{p} 110 \alpha$ cDNA with the CAAX motif at the $\mathrm{COOH}$ terminus was amplified in 293 cells, and viral stock solutions with a viral titer of $>10^{8} \mathrm{PFU} / \mathrm{ml}$ were prepared. 3T3-L1 adipocytes were infected with the vector by incubating the cells at a multiplicity of infection of 50 PFU/cell, as described previously [13, 14].

\section{Subcellular Fractionation}

The homogenates of 3T3-L1 adipocytes were subjected to subcellular fractionation to isolate low-density microsomes (LDM) and cytosol, as described previously [14]. These fractions were adjusted to a final protein concentration of 1 to $3 \mathrm{mg} / \mathrm{ml}$ and stored at $-80{ }^{\circ} \mathrm{C}$.

\section{Immunoblotting}

Proteins in whole cell lysates and subcellular fractions of 3T3-L1 adipocytes were prepared and electrophoresed on
7.5\% SDS-PAGE, and transferred to polyvinylidene difluoride membranes (Millipore, Bedford, MA), as described previously [14]. For detection of IRS-1, phosphoAkt and phospho-Ser-IRS-1, the membranes were blocked with TBS-T (10 mM Tris [pH 7.6], $150 \mathrm{mM} \mathrm{NaCl}, 0.1 \%$ Tween 20) containing 5\% nonfat dry milk, and incubated with the indicated antibody at $4{ }^{\circ} \mathrm{C}$ overnight. After washing, the membranes were incubated with HRP-conjugated secondary antibody. For detection of phosphotyrosine, the membranes were blocked with TBS-T containing 4\% bovine serum albumin (BSA) and incubated with PY20H at $4{ }^{\circ} \mathrm{C}$ overnight. The proteins were visualized with enhanced chemiluminescence reagents according to the manufacture's protocol (Amersham Pharmacia Biotech). The intensities of blots were quantified with a scanning densitometer.

\section{Phosphatidylinositol (PI) 3-Kinase Assay}

3T3-L1 adipocytes grown in $10 \mathrm{~cm}$ dishes were solubilized in lysis buffer $(20 \mathrm{mM}$ Tris [pH 7.6], $137 \mathrm{mM}$ $\mathrm{NaCl}, 1 \mathrm{mM} \mathrm{MgCl}, 1 \mathrm{mM} \mathrm{CaCl} 2,0.1 \mathrm{mM} \mathrm{Na}_{3} \mathrm{VO}_{4}, 1 \%$ Nonidet-P40, 10\% glycerol, 1 mM PMSF, $1 \mathrm{mM}$ dithiothreitol (DTT), and $0.1 \mathrm{mg}$ of aprotinin per $\mathrm{ml}$ ). The cell lysates were immunoprecipitated with anti-IRS-1 antibody for $2 \mathrm{~h}$ at $4{ }^{\circ} \mathrm{C}$, and then the immune complexes were collected by incubation with protein $\mathrm{G}$ Sepharose for 1 $\mathrm{h}$ at $4{ }^{\circ} \mathrm{C}$. PI3-kinase activity in the immunoprecipitates was determined, as previously described $[18,19] .{ }^{32} \mathrm{P}$ incorporated into phosphatidylinositol was visualized by autoradiography and quantified using Fuji BAS 2000 (Fuji Film, Tokyo, Japan).

\section{2-Deoxyglucose (DOG) Uptake in Response to Insulin Re-Stimulation}

After serum starvation for $3 \mathrm{~h}, 3 \mathrm{~T} 3$-L1 adipocytes were pretreated with the indicated inhibitors for $30 \mathrm{~min}$ and exposed to $20 \mathrm{nM}$ insulin for $8 \mathrm{~h}$. The cells were washed with PBS once, with Krebs-Ringer phosphate (KRP)-HEPES (10 mM HEPES, $131.2 \mathrm{mM} \mathrm{NaCl}, 4.7 \mathrm{mM} \mathrm{KCl}, 1.2 \mathrm{mM}$ $\left.\mathrm{MgSO}_{4}, 2.5 \mathrm{mM} \mathrm{CaCl}_{2}, 2.5 \mathrm{mM} \mathrm{NaH}_{2} \mathrm{PO}_{4}\right) 4$ times, and then incubated in the same buffer with $1 \%$ BSA ( $\mathrm{pH} 7.4)$ for 30 min, followed by re-stimulation with $20 \mathrm{nM}$ insulin for 15 min. Unlabeled and labeled 2-DOG $(0.1 \mathrm{mM}, 0.74 \mathrm{kBq} / \mathrm{well})$ were added to the cells for $4 \mathrm{~min}$. The reaction was stopped by adding $10 \mu \mathrm{M}$ of cytochalasin B (Sigma, St Louis, MO) and washing the cells with ice-cold PBS three times. The cells were solubilized in $1 \mathrm{ml}$ of $0.2 \%$ SDS and $0.2 \mathrm{~N}$ $\mathrm{NaOH}$. The radioactivity was quantified in a liquid scintillation counter.

\section{Statistical Analysis}

Data were analyzed by Student's $t$ test. $P$ values of $<0.05$ were considered statistically significant.

\section{RESULTS}

\section{Treatment with Either Lactacystin or Rapamycin Restores Insulin Signaling from IRS-1 to Akt Attenuated by Long Term Insulin Exposure}

To evaluate how mTOR-dependent serine/threonine phosphorylation and translocation of IRS-1 after long term insulin exposure affects the early step of insulin signals, we first compared the effects of either rapamycin, which inhibits serine/threonine phosphorylation and translocation of IRS-1 
to cytosol or lactacystin, which inhibits proteasomal degradation of IRS-1 [13, 14], on IRS-1 (Fig. 1A, B) and Akt (Fig. 1C, D). As we have previously described [13], prolonged insulin exposure for $4 \mathrm{~h}$ resulted in the degradation of IRS-1 (Fig. 1A), decreased tyrosine phosphorylation of IRS-1 (Fig. 1B) and decreased Akt

\section{A}
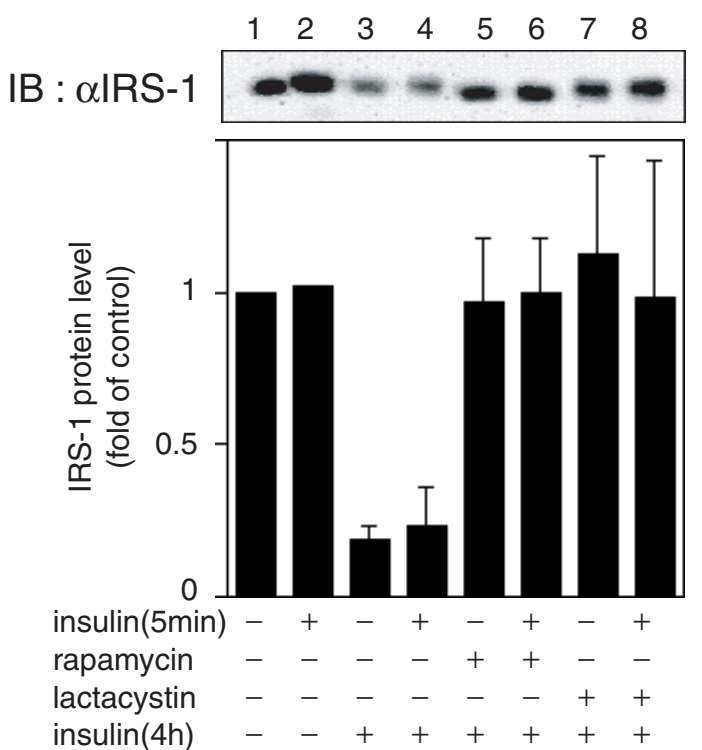

B

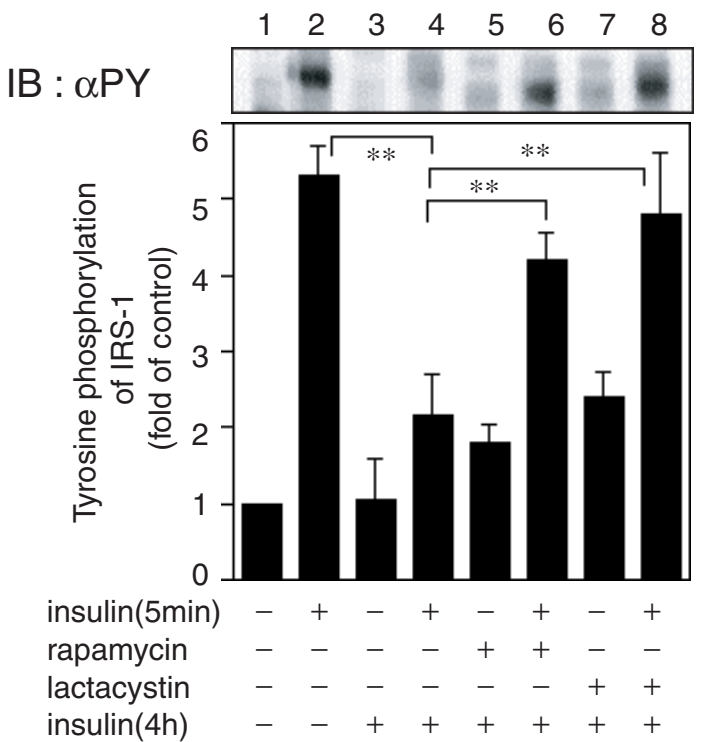

phosphorylation (Fig. 1C, D). Either rapamycin or lactacystin restored the protein levels (Fig. 1A) and tyrosinephosphorylation of IRS-1 (Fig. 1B) and Akt phosphorylation (Fig. 1C, D).

The effects of rapamycin or lactacystin on insulinstimulated PI3-kinase activity were next determined (Fig. 2).
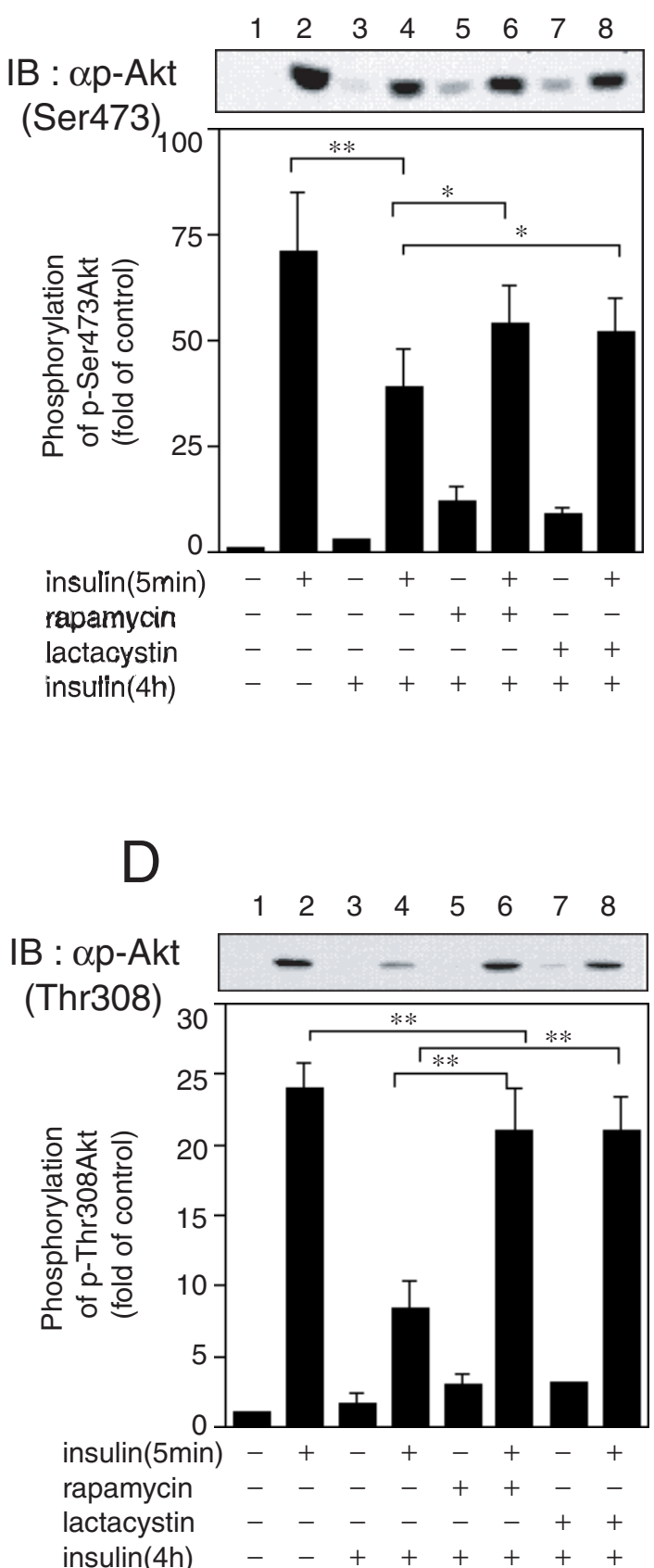

Fig. (1). Effect of the treatment with rapamycin or lactacystin during chronic insulin exposure of 3T3-L1 adipocytes on the levels of tyrosine-phosphorylated IRS-1 and Akt phosphorylation in response to insulin re-stimulation. 3T3-L1 adipocytes were serum-starved for $16 \mathrm{~h}$, incubated with vehicle $(0.1 \%$ DMSO), $20 \mathrm{nM}$ rapamycin or $10 \mu \mathrm{M}$ lactacystin for $30 \mathrm{~min}$. and then exposed to $20 \mathrm{nM}$ insulin for 4 $\mathrm{h}$. The cells were incubated with insulin-free medium for $30 \mathrm{~min}$. and then stimulated again with $20 \mathrm{nM}$ insulin for 5 min. Proteins were separated by SDS-PAGE and immunoblotted (IB) with A; anti-IRS-1 antibody ( $\alpha$ IRS-1), B; anti-phosphotyrosine antibody ( $\alpha$ PY) or

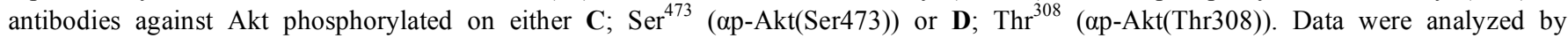
densitometry and expressed as fold increase compared with the values in control cells. Results are means $\pm \mathrm{SE}$ for three independent experiments $(*, P<0.05$; **, $P<0.01)$. 
Prolonged insulin exposure decreased the IRS-1-associated PI3-kinase activity in response to insulin re-stimulation (Fig. 2). Either rapamycin or lactacystin restored the response of IRS-1-associated PI3-kinase activity. Taken together, these findings suggested that IRS-1, which has already been serine/threonine phosphorylated and translocated into cytosol, has intact abilities to be tyrosine-phosphorylated and to activate PI3-kinase/ Akt pathway in response to insulin restimulation.

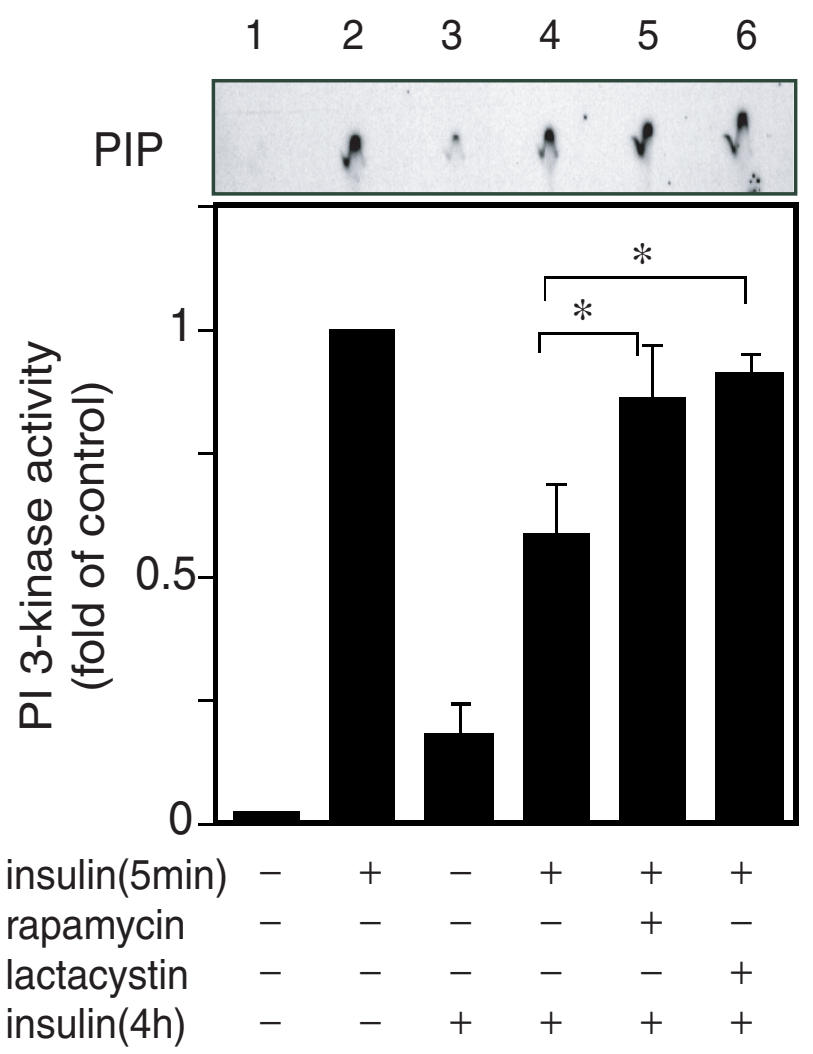

Fig. (2). Effect of the treatment with rapamycin or lactacystin during prolonged insulin exposure of 3T3-L1 adipocytes on IRS-1-associated PI 3-kinase activity in response to insulin restimulation. 3T3-L1 adipocytes were treated as in Fig. (1). Cell lysates were immunoprecipitated with anti-IRS-1 antibody, and the PI3-kinase activity present in the immunoprecipitates was assayed as described in "Materials and Methodology". Data were expressed as fold increase compared with the values for the acute insulin stimulation without prior prolonged insulin exposure. Results are means $\pm \mathrm{SE}$ for three independent experiments $(*, P<0.05)$.

Treatment with Either Lactacystin or Rapamycin Restores Insulin-Stimulated Tyrosine Phosphorylation of IRS-1 in p110 ${ }^{\text {CAAX }}$-Expressing Cells

We next examined the effects of rapamycin or lactacystin on insulin-stimulated tyrosine phosphorylation of IRS-1 in the cells transfected with adenovirus-mediated $\mathrm{p} 110^{\mathrm{CAAX}}$, a constitutively active form of regulatory subunit of PI3-kinase (Fig. 3). These cells were cultured in different concentrations of amino acids, because we have previously found that expression of $\mathrm{p} 110^{\mathrm{CAAX}}$ decreases IRS-1 protein in a concentration of amino acid-dependent manner [14]. The expression of $\mathrm{p} 110^{\mathrm{CAAX}}$ did not increase tyrosine phosphorylation of IRS-1. Four-fold excess of amino acids significantly decreased insulin-stimulated tyrosine phosphorylation of IRS-1, which was restored by the treatment with lactacystin or rapamycin (Fig. 3). These results support the notion that IRS-1, which is already serine/threonine phosphorylated and translocated to cytosol via the activation of PI3-kinase/ mTOR pathway, has an intact ability to be tyrosine phosphorylated.

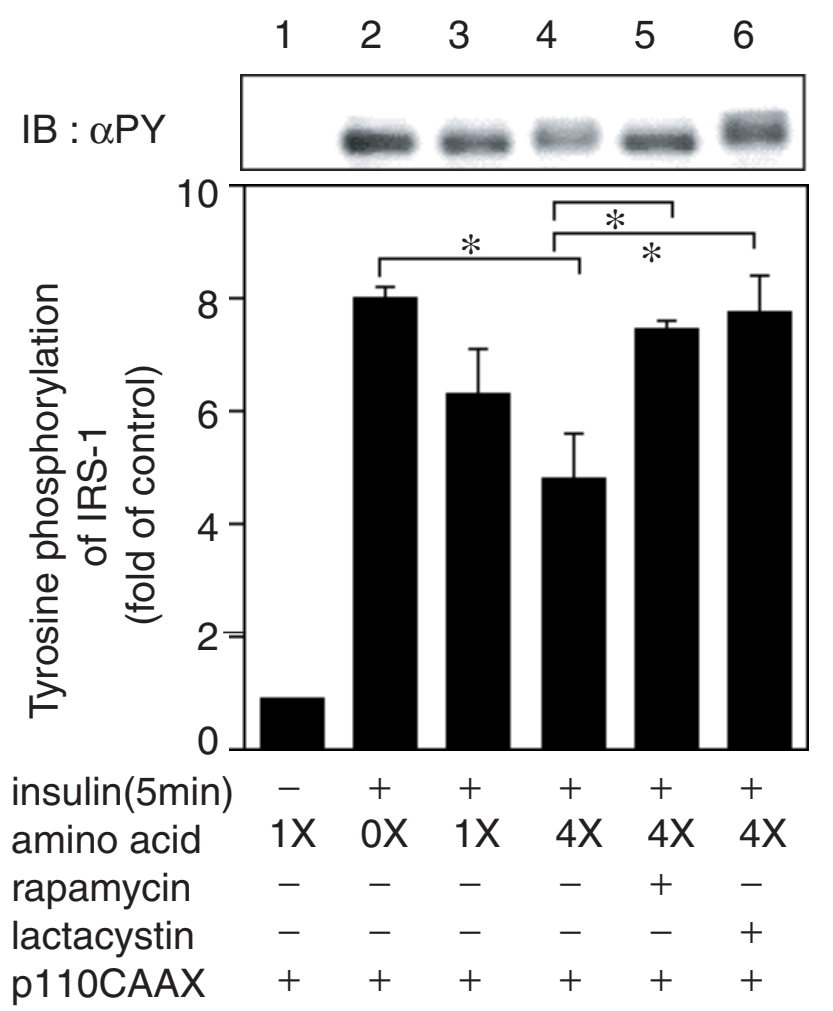

Fig. (3). Effect of the treatment with rapamycin or lactacystin on insulin-stimulated levels of tyrosine-phosphorylation of IRS1 in p110 ${ }^{\text {CAAX }}$-expressing 3T3-L1 adipocytes. 3T3-L1 adipocytes were infected with Ad5-p110 ${ }^{\text {CAAX }}$ at a multiplicity of infection of $50 \mathrm{PFU} /$ cell. At $48 \mathrm{~h}$ after infection, cells were incubated in serumfree MEM without amino acids (0X), with standard concentrations of amino acids (1X) or 4-fold excess of amino acids (4X) for $16 \mathrm{~h}$. During the same period, cells were treated with vehicle $(0.1 \%$ DMSO), $20 \mathrm{nM}$ rapamycin or $10 \mu \mathrm{M}$ lactacystin. Cells were then stimulated with $20 \mathrm{nM}$ insulin for $5 \mathrm{~min}$. Proteins were separated by SDS-PAGE and immunoblotted (IB) with anti-phosphotyrosine antibody $(\alpha \mathrm{PY})$. Data were analyzed by densitometry and expressed as fold increase compared with the values in control cells. Results are means $\pm \mathrm{SE}$ for three independent experiments $(*, P<0.05)$.

Treatment with Lactacystin During Prolonged Insulin Exposure Accumulates Serine-Phosphorylated IRS-1 in Cytosol

To further characterize the conditions of IRS-1 in the cells treated with lactacystin or rapamycin, we next determined intracellular localization of IRS-1 after $4 \mathrm{~h}$ insulin exposure (Fig. 4). As we recently reported [14], insulin treatment for $4 \mathrm{~h}$ decreased IRS-1 protein levels in both LDM and cytosol fractions. Rapamycin increased the protein levels of IRS-1, which were not serine phosphorylated, in both LDM and cytosol fractions. On the other hand, lactacystin treatment increased serine- 
phosphorylated IRS-1 mainly in cytosol fraction. These results suggest that the enhanced insulin signals in rapamycin-treated cells shown in Figs. $(\mathbf{1}, \mathbf{2})$ may be explained by the increased IRS-1 protein, which was not serine-phosphorylated. On the other hand, the accumulation of serine-phosphorylated IRS-1 in cytosol may be involved in the enhanced insulin signals in lactacystin-treated cells.
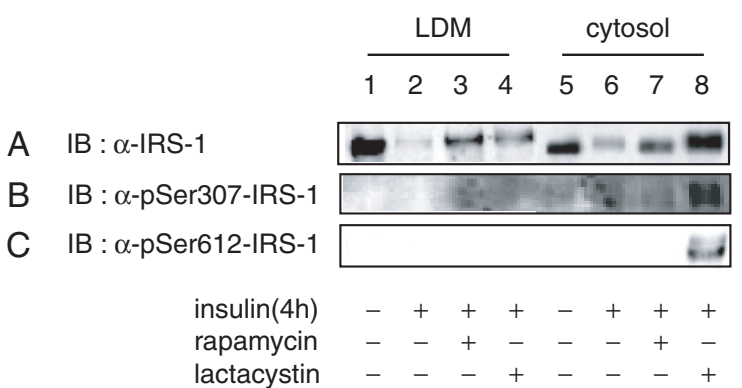

Fig. (4). Effect of the treatment with rapamycin or lactacystin during prolonged insulin exposure of 3T3-L1 adipocytes on the levels of serine-phosphorylation of IRS-1 in subcellular fractions. 3T3-L1 adipocytes were serum-starved for $16 \mathrm{~h}$, incubated with vehicle $(0.1 \%$ DMSO), $20 \mathrm{nM}$ rapamycin or $10 \mu \mathrm{M}$ lactacystin for $30 \mathrm{~min}$, and then exposed to $20 \mathrm{nM}$ insulin for $4 \mathrm{~h}$. The cells were incubated with insulin-free medium for $30 \mathrm{~min}$. Cells were homogenized and subjected to subcellular fractionation to yield the LDM and cytosol fractions. Proteins in each fraction were separated by SDS-PAGE and immunoblotted (IB) with $\mathbf{A}$; anti-IRS-1 antibody ( $\alpha$ IRS-1) or antibodies against IRS-1 phosphorylated on either B; $\operatorname{Ser}^{307}\left(\alpha\right.$-pSer307-IRS-1) or C; $\operatorname{Ser}^{612}$ $(\alpha$-pSer612-IRS-1). Results are the representative of three independent experiments.

Serine-Phosphorylated IRS-1 in the Cytosol Can Be Tyrosine-Phosphorylated in Response to Insulin ReStimulation

We next examined tyrosine-phosphorylation status of IRS-1 in both LDM and cytosol fractions in the cells treated with either rapamycin or lactacystin (Fig. 5). Rapamycin treatment during the prolonged insulin exposure increased the levels of tyrosine-phosphorylated IRS-1 in response to insulin re-stimulation both in the LDM and in the cytosol in parallel with the increased protein levels of IRS-1 in each fraction (Fig. 5A, B). In contrast, lactacystin increased the level of tyrosine-phosphorylated IRS-1 only in the cytosol, where most of IRS-1 protein existed (Fig. 5A, B). These results suggested that serine/threonine phosphorylation and the translocation of IRS-1 to cytosol during prolonged insulin exposure do not significantly affect the ability of IRS-1 to be tyrosine-phosphorylated in response to insulin re-stimulation.

\section{Rapamycin Partially Restores Glucose Transport During Prolonged Insulin Exposure, But Lactacystin Does Not}

We finally examined the ability of IRS-1, which was serine/threonine-phosphorylated and translocated to cytosol, to stimulate glucose transport in response to insulin restimulation (Fig. 6). As we have previously described [13, 14], after $4 \mathrm{~h}$ insulin exposure, the basal glucose uptake was too high to evaluate the response to short term insulin
A

$A$
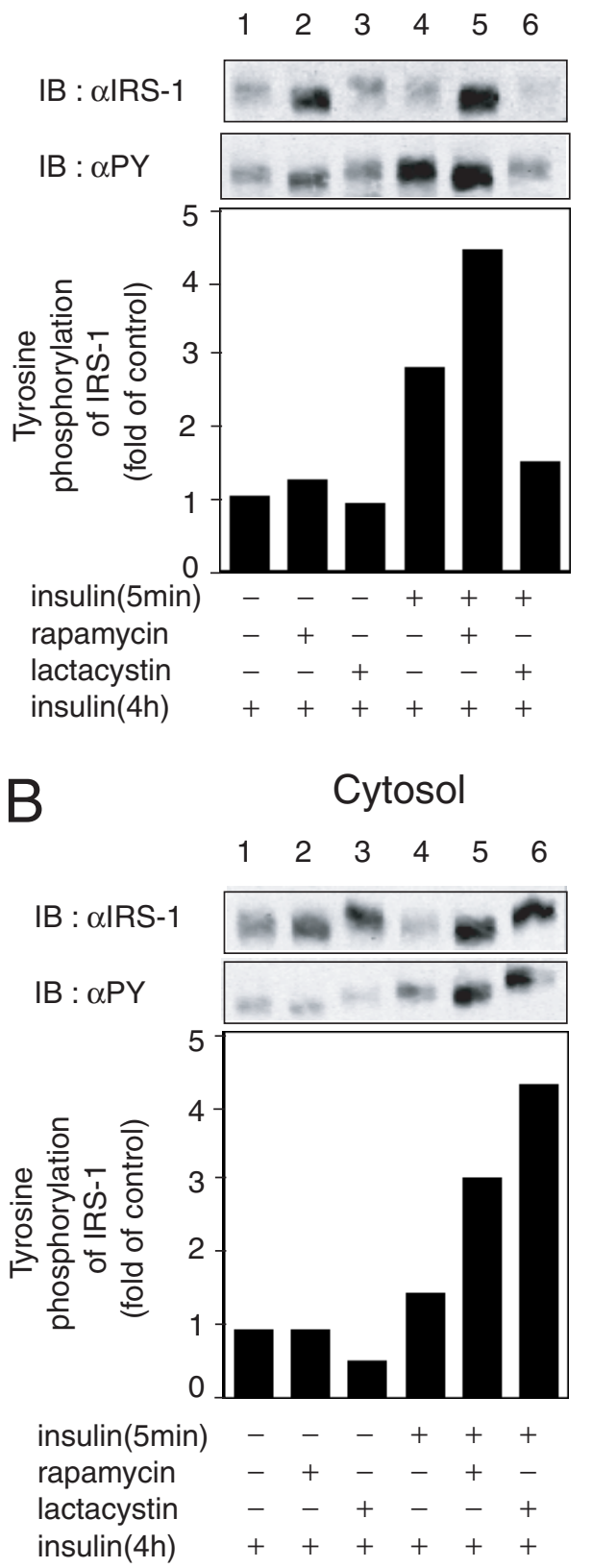

Fig. (5). Effect of the treatment with rapamycin or lactacystin during prolonged insulin exposure of 3T3-L1 adipocytes on the levels of tyrosine-phosphorylation of IRS-1 in subcellular fractions in response to insulin re-stimulation. 3T3-L1 adipocytes were serum-starved for $16 \mathrm{~h}$, incubated with vehicle (0.1\% DMSO), $20 \mathrm{nM}$ rapamycin or $10 \mu \mathrm{M}$ lactacystin for $30 \mathrm{~min}$. and then exposed to $20 \mathrm{nM}$ insulin for $4 \mathrm{~h}$. The cells were incubated with insulin-free medium for $30 \mathrm{~min}$, and then stimulated with 20 $\mathrm{nM}$ insulin for $5 \mathrm{~min}$. Cells were homogenized and subjected to subcellular fractionation to yield $\mathbf{A}$; the LDM and $\mathbf{B}$; the cytosol fractions. Proteins in each fraction were separated by SDS-PAGE and immunoblotted (IB) with anti-IRS-1 antibody ( $\alpha$ IRS-1) or antiphosphotyrosine antibody $(\alpha \mathrm{PY})$. The data for anti-phosphotyrosine antibody blots were expressed as fold increase compared with the values for each fraction in the cells treated with insulin for $4 \mathrm{~h}$ without acute insulin stimulation. Results are means for two independent experiments. 
re-stimulation. Therefore, we extended the insulin exposure period to $8 \mathrm{~h}$, when 2-deoxyglucose (DOG) uptake prior to insulin re-stimulation was significantly decreased (Fig. 6). The response of 2-DOG uptake by insulin re-stimulation for 15 min was significantly decreased after the prolonged insulin exposure for $8 \mathrm{~h}$. Treatment with rapamycin during prolonged insulin exposure partially but significantly increased the response of 2-DOG uptake. In contrast, lactacystin had no effect on the response (Fig. 6). These results suggest that the ability of IRS-1 to stimulate glucose transport in response to insulin re-stimulation is attenuated at least in part by the serine/threonine phosphorylation and the translocation to cytosol.

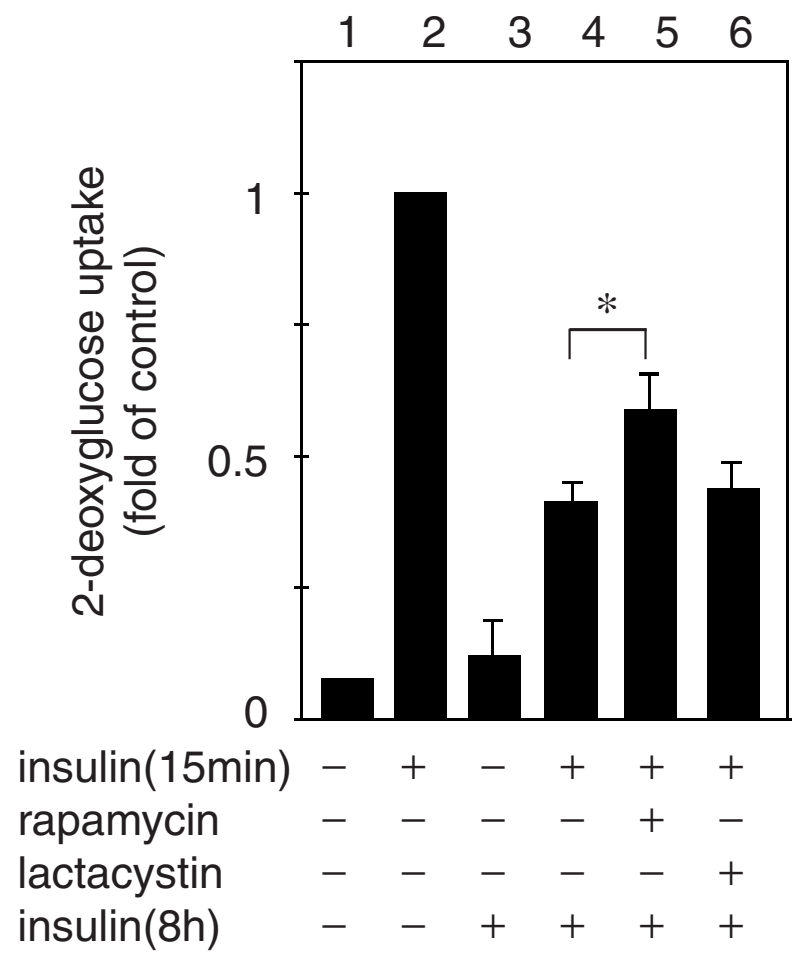

Fig. (6). Effect of the treatment with rapamycin or lactacystin during prolonged insulin exposure of 3T3-L1 adipocytes on 2deoxyglucose uptake in response to insulin re-stimulation. 3T3L1 adipocytes were serum-starved for $3 \mathrm{~h}$, incubated with vehicle (0.1\% DMSO), $20 \mathrm{nM}$ rapamycin or $10 \mu \mathrm{M}$ lactacystin for $30 \mathrm{~min}$. and exposed to $20 \mathrm{nM}$ insulin for $8 \mathrm{~h}$. The cells were washed once with PBS, four times with KRP-HEPES. Cells were then incubated in the same buffer containing $1 \%$ BSA for $30 \mathrm{~min}$, and stimulated again with $20 \mathrm{nM}$ insulin for $15 \mathrm{~min}$. 2-deoxy-D-[ $\left.{ }^{3} \mathrm{H}\right]$ glucose uptake was measured as described in "Materials and Methods". Data were expressed as the ratio compared with the values for acute insulin stimulation without prior prolonged insulin exposure. Results are means \pm SE for four independent experiments (*, $P<0.05)$

\section{DISCUSSION}

We have previously shown that activation of mTOR pathway during prolonged insulin stimulation negatively regulates insulin signals by modulating serine/threonine phosphorylation, subcellular localization and protein stability of IRS-1. We have also identified the novel function of mTOR which integrates nutrient availability and metabolic actions of insulin $[13,14]$. Recent studies in mouse embryo fibroblasts deficient in tuberous sclerosis complex (TSC) proteins, which are negative regulators of mTOR pathway, supported our previous conclusions. They showed that constitutive activation of mTOR pathway decreased response to insulin through modulating IRS serine/threonine phosphorylation, protein stability and/or expression [15, 16]. Furthermore, in insulin target tissues of genetic and diet-induced animal models of insulin resistance, markedly elevated activity of S6K1, a downstream effecter of mTOR, and increased serine phosphorylation of IRS-1 were observed [17]. Moreover, the deletion of S6K1 gene in mice protected against high-fat dietinduced insulin resistance [17]. Thus, it was recognized that the mTOR-dependent effects on IRS proteins might be involved in the pathogenesis of insulin resistance in obesity and type 2 diabetes $[20,21]$. Based on these findings, the mTORdependent feedback loop would be a candidate target for the treatment of patients with insulin resistance.

One of the novel findings in the present study is that the IRS-1, which has already been phosphorylated on serine/threonine residues and translocated into the cytosol by the activation of mTOR-dependent pathway during prolonged insulin exposure, has an intact ability to be tyrosinephosphorylated upon insulin re-stimulation. We also found that the cytosolic IRS-1, phosphorylated on multiple serine residues, has an intact ability to activate PI3-kinase pathway leading to activation of Akt in response to insulin re-stimulation. Thus, inhibition of IRS-1 degradation by lactacystin during chronic insulin exposure resulted in accumulation of IRS-1 in the cytosol, which was phosphorylated on multiple residues including $\mathrm{Ser}^{307}$ and $\mathrm{Ser}^{612}$. In this condition, tyrosine phosphorylation of IRS-1, IRS-1-associated PI 3-kinase activity and Akt phosphorylation observed after insulin re-stimulation were restored as much as the effects of rapamycin, which inhibited serine/threonine phosphorylation and translocation of IRS-1. As shown in Fig. (3), lactacystin increased the insulinstimulated tyrosine-phosphorylation of IRS-1 to an extent similar to the effect of rapamycin in $\mathrm{p} 110^{\mathrm{CAAX}}$-expressing cells. We recently reported that constitutive activation of PI3-kinase pathway by $\mathrm{p} 110^{\mathrm{CAAX}}$ in combination with high concentrations of amino acids continuously induced the mTOR-dependent effects on IRS-1 [14]. These results further supported the idea that serine/threonine phosphorylation and translocation of IRS-1 to cytosol has an intact ability to be tyrosine-phosphorylated. It should be noted that, unlike the case of prolonged insulin exposure, activation of PI3-kinase pathway by $\mathrm{p} 110^{\mathrm{CAAX}}$ elicits the effects of mTOR on IRS-1 without inducing its tyrosine phosphorylation $[13,14]$. These results eliminate the possibility that the residual tyrosine phosphorylation of IRS- 1 after chronic insulin exposure affected the subsequent response to insulin restimulation.

Our recent studies have revealed that serine phosphorylation of IRS-1 follows its tyrosine phosphorylation in insulinstimulated 3T3-L1 adipocytes. During the insulin stimulation period, e.g. from $20 \mathrm{~min}$ to $60 \mathrm{~min}$, phosphoylation was observed on both tyrosine and serine/threonine residues at the same time [13]. Furethermore, even after IRS-1 was serine phosphorylated by the pre-treatment with TNF $\alpha$ or IL-1, short term insulin treatment could induce tyrosine phosphorylation $[22,23]$. These results clearly demonstrate that there may exist both tyrosine-phosphorylated IRS-1 and serine- phosphorylated IRS-1 in a cell. However, IRS-1 is possibly tyrosine- and serine- 
phosphorylated in a cell in the following different ways, i.e., both tyrosine and serine residues are phosphorylated on all the IRS-1 protein. Alternatively, some part of IRS-1 protein is serine-phorphorylated and the residual IRS- 1 is tyrosine phosphorylated, which may exist in the different sub-cellular fraction and may not be serine-phosphorylated. It is important to distinguish these phosphorylation conditions of IRS-1. Coimmunoprecipitation experiment using anti-phosphotyrosine antibody and anti-phosphoserine antibody may be useful to clarify this phenomenon. Unfortunately, we could not perform those experiments, which would be addressed in the near future.

Is serine-phosphorylated IRS-1 in LDM fraction of rapamycin-treated cells resistant to proteosomal degradation compared to cytosolic IRS-1? We have reported that lactasystin almost completely inhibits degradation of IRS-1 in 3T3-L1 adipocytes, suggesting that degradation of IRS-1 is highly dependent on ubiquitin-proteasomal system. Because proteasome exists in cytosol, proteins should be translocated from the other fractions to cytosol fraction for the proteasomal dagradation. Therefore, it is less likely that IRS- 1 protein in LDM fraction is degraded using ubiquitin-proteasome system, and the translocation of IRS-1 to cytosol fraction is important for the degradation.

Serine phosphorylation of IRS-1 has been implicated in the mechanism of the impaired response to insulin induced by various candidate mediators of insulin resistance such as tumor necrosis factor (TNF)- and free fatty acids (FFA) [24-26]. For example, TNF- $\alpha$ activates c-jun $\mathrm{NH}_{2}$-terminal kinase (JNK), which promotes phosphorylation of IRS-1 on $\operatorname{Ser}^{307}[24,27]$. FFA as well as TNF- has been reported to stimulate the inhibitor $\mathrm{\kappa B}$ kinase (IKK), which may also be responsible for the IRS-1 phosphorylation including $\mathrm{Ser}^{307}$ [25]. In contrast to our results, these studies reported that phosphorylation of IRS-1 on $\mathrm{Ser}^{307}$ impairs the ability of IRS- 1 to be tyrosinephosphorylated in response to insulin stimulation, thereby inhibits downstream insulin signals. Different experimental or cellular conditions may at least in part account for the discrepancy. For example, in the cells such as 3T3-L1 adipocytes, which express high levels of IRS-1 and insulin receptor, the phosphorylation of $\mathrm{Ser}^{307}$, which compromises the function of PTB domain of IRS-1 [24, 27], may not be sufficient to significantly disturb the interaction of IRS-1 with insulin receptor [28] especially when the cells are stimulated with high concentration of insulin. Therefore, we do not exclude the possibility that the response of IRS-1 tyrosine phosphorylation may be impaired to some extent, when the cells are re-stimulated with submaximal concentrations of insulin. Alternatively, activation of mTOR pathway may elicit phosphorylation of IRS-1 serine/threonine residues, which are different from those phosphorylated via other pathways such as JNK. The effects of serine/threonine phosphorylation on tyrosine phosphorylation and transmition of insulin signals may be different among the different serine/threonine residues. Indeed, the extent of electrophoretic mobility shift of IRS-1 induced by insulin stimulation is much greater than that induced by JNK activation by anisomycin (data not shown). These results suggest that more serine/threonine residues may be phosphorylated in response to activation of mTOR pathway, compared with the residues phosphorylated in response to JNK activation. Therefore, it would be probable that phosphorylation of serine/threonine residues specifically induced by acitivation of mTOR pathway might increase the ability of IRS-1 to be tyrosine-phsophorylated, thereby overcoming the inhibitory effects of other phosphorylated residues such as $\mathrm{Ser}^{307}$, as several reports indicated that phosphorylation of certain serine residues of IRS-1 may increase insulin sensitivity [29-31].

Another remarkable finding in this study is that, despite the intact abilities of the cytosolic IRS-1 phosphorylated on multiple serine residues to be tyrosine-phosphorylated and to activate PI 3-kinase pathway including Akt, this does not seem to lead to stimulation of glucose transport. The increase of glucose transport in response to insulin re-stimulation appears to be dependent upon the intracellular localization of the tyrosinephosphorylated IRS-1 observed after insulin re-stimulation. This is because rapamycin increased the tyrosinephosphorylated IRS-1 observed after insulin re-stimulation not only in the cytosol but also in the LDM, and partially but significantly restored the response of glucose transport. On the other hand, lactacystin increased the tyrosine-phosphorylated IRS-1 mainly in the cytosol and had no effect on the response of glucose transport, despite the increased activation of IRS-1associated PI 3-kinase and Akt. The partial recovery of the response of glucose transport by rapamycin is consistent with the report by others [32], and at least part of the incompleteness of the recovery would probably be accounted for by the mTORindependent down-regulation of other molecules involved in glucose transport during chronic insulin exposure. Although S6K-dependent supression of IRS-1 gene expression has been reported [15], it is unlikely that the effect of rapamycin was due to recovery of IRS-1 gene expression. Inhibition of proteasomal degradation of IRS-1 by lactacystin inhibited the decrease of IRS-1 protein level during the $8 \mathrm{~h}$ insulin exposure as much as the effect of rapamycin (data not shown). Taken together, the ability of IRS-1 to stimulate glucose transport seems to be attenuated as a result of mTOR-dependent IRS-1 translocation independently of the ability of the IRS-1 to be tyrosinephosphorylated and to activate PI3-kinase pathway. The mTOR-dependent IRS-1 translocation may result in impaired PI $(3,4,5) \mathrm{P}_{3}$ production in a particular intracellular compartment in response to insulin re-stimulation and possibly attenuate the activity of specific Akt isoform and/or other molecules such as $\mathrm{PKC} \lambda / \zeta$ involved in stimulation of GLUT4 translocation, thereby attenuating the response of glucose transport. Future studies are required to clarify these issues.

\section{CONCLUSION}

In conclusion, the results of the present study indicate that, in 3T3-L1 adipocytes, the mTOR-dependent phosphorylation of multiple serine/threonine residues and translocation of IRS-1, do not remarkably affect the abilities of IRS-1 to be tyrosinephosphorylated and to activate IRS-1-associated PI3-kinase and downstream Akt. Nevertheless, the ability to stimulate glucose transport is attenuated likely through the mTOR-dependent subcellular redistribution of IRS-1. Therefore, altered localization of IRS proteins in insulin target tissues may contribute to the molecular mechanism of insulin resistance induced by the activation of mTOR pathway, which is possibly associated with the pathophysiology of some diseases, such as obesity and type 2 diabetes.

\section{ACKNOWLEDGMENTS}

We are grateful to Prof. Jerrold M. Olefsky (University of Clifornia, San Diego) for the generous gift of adenovirus constructs. Drs. Shiho Fujisaka, Hikari Suzuki, Yu Yamazaki, 
Satoko Senda, Akiko Takikawa, Ken Ishizuka, and Manabu Ishiki (University of Toyama) are acknowledged for the technical assistance. Drs. Yasumitsu Takata, Toshiyasu Sasaoka, Katsuya Yamazaki, Masaharu Urakaze (University of Toyama) and Takeshi Imamura (Shiga University of Medical Science) are acknowledged for the useful discussion. This study was supported by a grant-in-aid for Scientific Research from the Ministry of Education, Science, Sports and Culture, Japan (10671060 to T.H.).

\section{REFERENCES}

[1] Haruta T, Morris AJ, Rose DW, Nelson JG, Mueckler M, Olefsky JM. Insulin-stimulated GLUT4 translocation is mediated by a divergent intracellular signaling pathway. J Biol Chem 1995; 270: 27991-4.

[2] Martin SS, Haruta T, Morris AJ, Klippel A, Williams LT, Olefsky JM. Activated phosphatidylinositol 3-kinase is sufficient to mediate actin rearrangement and GLUT4 translocation. J Biol Chem 1996; 271: $17605-8$

[3] Hotamisligil GS, Peraldi P, Budvari A, Ellis RW, White MF, Spiegelman BM. IRS-1-mediated inhibition of insulin receptor tyrosine kinase activity in TNF- $\alpha$ - and obesity-induced insulin resistance. Science 1996; 271: 665-8.

[4] Liu Y-F, Paz K, Hersckovitz A, et al. Insulin stimulates PKC mediated phosphorylation of insulin receptor substrate-1 (IRS-1). A self-attenuated mechanism to negatively regulate the function of IRS proteins. J Biol Chem 2001; 276: 14459-65.

[5] Staubs PA, Nelson JG, Reichart DR, Olefsky JM. Platelet-derived growth factor inhibits insulin stimulation of insulin receptor substrate-1-associated phosphatidylinositol 3-kinase in 3T3-L1 adipocytes without affecting glucose transport. J Biol Chem 1998; 273: 25139-47.

[6] Mothe I, Van Obberghen E. Phosphorylation of insulin receptor substrate-1 on multiple serine residues, $612,632,662$, and 731 , modulates insulin action. J Biol Chem 1996; 271: 11222-7.

[7] DeFea K, Roth RA. Modulation of insulin receptor substrate-1 tyrosine phosphorylation and function by mitogen-activated protein kinase. J Biol Chem 1997; 272: 31400-6.

[8] Li J, DeFea K, Roth RA. Modulation of insulin receptor substrate-1 tyrosine phosphorylation by an Akt/phosphatidylinositol 3-kinase pathway. J Biol Chem 1999; 274: 9351-6.

[9] Kanety H, Feinstein R, Papa MZ, Hemi R, Karasik A. Tumor Necrosis Factor $\alpha$-induced phosphorylation of insulin receptor substrate-1 (IRS-1). J Biol Chem 1995; 270: 23780-4.

[10] Tanti JF, Greumeaux T, Van Obberghen E, Le Marchand-Brustel Y. Serine/threonine phosphorylation of insulin receptor substrate-1 modulates insulin receptor signaling. J Biol Chem 1994; 269: 605157.

[11] Jullien D, Tanti JF, Heydrick SJ, et al. Differential effects of okadaic acid on insulin-stimulated glucose and amino acid uptake and phosphatidylinositol 3-kinase activity. J Biol Chem 1993; 268: 15246-51.

[12] Clark SF, Molero JC, James DE. Release of insulin receptor substrate proteins from an intracellular complex coincides with the development of insulin resistance. J Biol Chem 2000; 275: 381926.

[13] Haruta $\mathrm{T}$, Uno $\mathrm{T}$, Kawahara $\mathrm{J}$, et al. A rapamycin-sensitive pathway down-regulates insulin signaling via phosphorylation and proteosomal degradation of insulin receptor substrate-1. Mol Endocrinol 2000; 14: 783-94.

[14] Takano A, Usui I, Haruta T, et al. Mammalian target of rapamycin pathway regulates insulin signaling via subcellular redistribution of insulin receptor substrate- 1 and integrates nutritional signals and metabolic signals of insulin. Mol Cell Biol 2001; 21: 5050-62.

[15] Harrington LS, Findlay GM, Gray A, et al. The TSC1-2 tumor suppressor controls insulin-PI3K signaling via regulation of IRS proteins. J Cell Biol 2004; 166: 213-23.

[16] Shah OJ, Wang Z, Hunter T. Inappropriate activation of the TSC/Rheb/mTOR/S6K cassette induces IRS1/2 depletion, insulin resistance, and cell survival deficiencies. Curr Biol 2004; 14: 16506.

[17] Um SH, Frigerio F, Watanabe M, et al. Absence of S6K1 protects against age- and diet-induced obesity while enhancing insulin sensitivity. Nature 2004; 431: 200-5.

[18] Iwata M, Haruta T, Usui I, et al. Pioglitazone ameliorates tumor necrosis factor- $\alpha$-induced insulin resistance by a mechanism independent of adipogenic activity of peroxisome proliferator-activated receptor- $\gamma$. Diabetes 2001; 50: 1083-92.

[19] Takano A, Haruta T, Iwata M, et al. Growth hormone induces cellular insulin resistance by uncoupling phosphatidylinositol 3kinase and its downstream signals in 3T3-L1 adipocytes. Diabetes 2001; 50: 1891-900.

[20] Manning BD. Balancing Akt with S6K: implications for both metabolic diseases and tumorigenesis. J Cell Biol 2004; 167: 399403

[21] Harrington LS, Findlay GM, Lamb RF. Restraining PI3K: mTOR signalling goes back to the membrane. Trends Biochem Sci 2005 30: $35-42$.

[22] He J, Usui I, Ishizuka K, et al. Interleukin-1 $\alpha$ Inhibits Insulin Signaling with Phosphorylating Insulin Receptor Substrate-1 on Serine Residues in 3T3-L1 Adipocytes. Mol Endocrinol 2006; 20: $114-24$

[23] Ishizuka K, Usui I, Kanatani Y, et al. Chronic TNF $\alpha$ Treatment Causes Insulin Resistance via IRS-1 Serine Phosphorylation and SOCS3 Induction in 3T3-L1 Adipocytes. Endocrinology 2007; 148: 2994-3003

[24] Rui L, Aguirre V, Kim JK, et al. Insulin/IGF-1 and TNF- $\alpha$ stimulate phosphorylation of IRS-1 at inhibitory Ser307 via distinct pathways. J Clin Invest 2001; 107: 181-9.

[25] Gao Z, Hwang D, Bataille F, et al. Serine phosphorylation of insulin receptor substrate 1 by inhibitor $\mathrm{\kappa B}$ kinase complex. J Biol Chem 2002; 277: 48115-21.

[26] Yu C, Chen Y, Cline GW, et al. Mechanism by which fatty acids inhibit insulin activation of insulin receptor substrate-1 (IRS-1)associated phosphatidylinositol 3-kinase activity in muscle. J Biol Chem 2002; 277: 50230-6.

[27] Aguirre V, Uchida T, Yenush L, Davis R, White MF. The c-Jun $\mathrm{NH} 2$-terminal kinase promotes insulin resistance during association with insulin receptor substrate-1 and phosphorylation of Ser307. J Biol Chem 2000; 275: 9047-54.

[28] Yenush L, Makati KJ, Smith-Hall J, Ishibashi O, Myers Jr-MG, White MF. The pleckstrin homology domain is the principle link between the insulin receptor and IRS-1. J Biol Chem 1996; 271: 24300-6.

[29] Paz K, Liu YF, Shorer H, et al. Phosphorylation of insulin receptor substrate-1 (IRS-1) by protein kinase B positively regulates IRS-1 function. J Biol Chem 1999; 274: 28816-22.

[30] Jakobsen SN, Hardie DG, Morrice N, Tornqvist HE. 5'-AMPactivated protein kinase phosphorylates IRS-1 on Ser-789 in mouse $\mathrm{C} 2 \mathrm{C} 12$ myotubes in response to 5-aminoimidazole-4-carboxamide riboside. J Biol Chem 2001; 276: 46912-6.

[31] Giraud J, Leshan R, Lee YH, White MF. Nutrient-dependent and insulin-stimulated phosphorylation of insulin receptor substrate-1 on serine 302 correlates with increased insulin signaling. J Biol Chem 2004; 279: 3447-54.

[32] Berg CE, Lavan BE, Rondinone CM. Rapamycin partially prevents insulin resistance induced by chronic insulin treatment. Biochem Biophys Res Commun 2002; 293: 1021-7. 